\title{
Artigo/Article
}

\section{Prevalência da infecção pelo Schistosoma mansoni em dois municípios do Estado de Alagoas}

\author{
Prevalence of Schistosoma mansoni infection in two municipalities of the State of Alagoas, \\ Brazil
}

\author{
Danylo César Correia Palmeira ${ }^{1}$, Adriano Gonçalves de Carvalho ${ }^{1}$, Katyane Rodrigues ${ }^{2}$ e Janira Lúcia \\ Assumpção Couto ${ }^{3}$
}

\begin{abstract}
RESUMO
Introdução: Estudos preliminares indicam uma provável expansão da esquistossomose em Alagoas. Este trabalho analisa a ocorrência do Schistosoma mansoni em escolares de dois municípios, localizados nas bacias hidrográficas dos rios Mundaú e Paraíba. Métodos: O grupo de estudo foi constituído por 690 escolares da zona urbana, com idade entre sete e 15 anos. Foram aplicados interrogatórios socioeconômicos e foram usados exames parasitológicos para diagnostico da esquistossomose (métodos de Kato e de Lutz). Foram tratados os casos positivos e foram ministradas palestras para alunos e familiares sobre educação sanitária. Resultados: A prevalência de Schistosoma mansoni foi de 24,9\%. A associação entre esquistossomose e outras parasitoses foi significativa em relação à tricuríase $(p<0,05)$. Apenas $50 \%$ dos infectados residem em domicílios de alvenaria com saneamento e abastecimento d'água por rede pública. Entre os doentes, $48,1 \%$ costumam ir frequentemente às coleções aquáticas e 55,8\% deles conhecem moluscos nos rios. A análise de concordância entre os métodos de Lutz e do Kato mostrou discordância em 54,2\%, com maior sensibilidade para o de Lutz (76,4\%). Conclusões: Os índices obtidos caracterizam os municípios como zonas hiperendêmicas, porque taxas de prevalência acima de 5\% são consideradas altas de acordo com a Organização Mundial de Saúde. A elevada prevalência aqui encontrada foi também duas vezes maior que a apresentada pelo Programa Brasileiro de Controle da Esquistossomose.

Palavras-chaves: Esquistossomose. Escolares. Alagoas. Diagnóstico.
\end{abstract}

\begin{abstract}
Introduction: Preliminary studies indicate a probable schistosomiasis expansion in Alagoas. This work analyzes the occurrence of Schistosoma mansoni in students from two municipalities, located in the Mundaú and Paraíba rivers basins. Methods: The study group consisted of 690 students in the urban area, aged between seven and 15 years. A standardized socio-economic questionnaire was filled out and parasitological tests were used to diagnosis schistosomiasis (Lutz and Kato methods). Positive cases were treated and students and their families were lectured about health education. Results: The Schistosoma mansoni prevalence was $24.9 \%$. The association between schistosomiasis and other parasitic diseases was significant in relation to trichuriasis $(\mathrm{p}<0.05)$. Only 50\% of infected children lived in brick homes with sanitation and public water supply. Among them, $48.1 \%$ used to go usually to the water collections and $55.8 \%$ of them knew rivers snails. The concordance analysis between the Lutz and Kato methods showed disagreement in $54.2 \%$, with greater sensitivity for the Lutz technique (76.4\%). Conclusions: The indices obtained characterize the municipalities as hyperendemic areas, because prevalence rates over $5 \%$ are considered high according to the World Health Organization. The high prevalence observed here is also two times higher than that reported by the Brazilian Schistosomiasis Program Control.
\end{abstract}

Key-words: Schistosomiasis. Students. Alagoas. Diagnosis.

\footnotetext{
1. Faculdade de Medicina, Universidade Federal de Alagoas, Maceió, AL. 2. Escola de Enfermagem e Farmácia, Universidade Federal de Alagoas, Maceió, AL. 3. Setor de Parasitologia, Instituto de Ciências Biológicas e da Saúde, Universidade Federal de Alagoas, Maceió, AL.

Endereço para correspondência: Dr. Danylo César Correia Palmeira. R. Tenente José Ferreira de Souza 12/Quadra 03. Conjunto Santo Eduardo, Poço, 57025-315 Maceió, AL.

e-mail: danylo_palmeira@hotmail.com

Recebido para publicação em 12/11/2009

Aceito em 23/02/2010
}

\section{INTRODUÇÃO}

A esquistossomose mansônica, causada pelo trematódeo Schistosoma mansoni Sambon, 1907, ainda constitui moléstia de elevada prevalência nos países em desenvolvimento, notadamente o Brasil ${ }^{1}$. Uma vez que se associa à pobreza e aos hábitos culturais de uma determinada população, representa um importante indicativo do nível socioeconômico ${ }^{2}$.

No Brasil, cerca de 6,3 milhões de indivíduos são portadores da forma intestinal da doença, a maioria oriunda da região Nordeste, o que evidencia o deficiente saneamento básico e má higienização sanitária da população ${ }^{3}$.

A confirmação diagnóstica da esquistossomose é feita, principalmente, pela simples análise de amostras fecais. As técnicas de concentração apresentam maior sensibilidade e, consequentemente, são mais utilizadas para confirmação de esquistossomose e outras parasitoses ${ }^{4}$.

A técnica de Lutz (1919), de sedimentação simples, é mais usada na rotina ${ }^{5}$. A carga parasitária de um paciente, fator determinante da forma hepatoesplênica, pode ser calculada através do método quantitativo de Kato, modificado por Katz (1972), que estima o número de ovos por grama de fezes (opg) com elevada sensibilidade e simplicidade ${ }^{6}$.

Estudos realizados pela Fundação Nacional de Saúde (FUNASA, 1995) evidenciaram que, entre 18 estados do Nordeste e Sudeste, o mais elevado índice de exames parasitológicos positivos foi encontrado na população alagoana. Também, foi constatado que cerca de $60 \%$ do território do estado seria endêmico e que aproximadamente dois milhões de pessoas estariam expostas à infecção 7 . Além disso, o molusco Biomphalaria glabrata Say, 1818, principal espécie transmissora, foi identificado em áreas da lavoura canavieira alagoana ${ }^{8,9}$. 
Atualmente, 72 municípios compõem a chamada faixa de risco da esquistossomose. De acordo com a FUNASA (1995), uma provável expansão da endemia acontece em municípios alagoanos inseridos nas bacias hidrográficas dos rios Mundaú e Paraíba ${ }^{7,10,11}$.

Crianças em idade escolar constituem o grupo com maior vulnerabilidade e exposição a parasitoses, por isso a Organização Mundial de Saúde (OMS) estabelece sua prioridade nos programas de controle das endemias ${ }^{12,13}$. Elas também estão mais expostas à esquistossomose pelo maior contato com a água em atividades recreativas nos rios ${ }^{2,12}$.

Este estudo teve como objetivos analisar a ocorrência da esquistossomose mansônica em escolares de municípios banhados pelos rios Mundaú e Paraíba.

\section{MÉTODOS}

Trata-se de um levantamento epidemiológico analítico e observacional, realizado em parceria com as secretarias municipais de saúde de cada município, com a autorização da direção escolar de cada unidade educacional investigada. Foi executado após aprovação do Comitê de Ética em Pesquisa da Universidade Federal de Alagoas.

\section{Área de estudo}

A pesquisa foi realizada em dois municípios alagoanos, localizados nas bacias hidrográficas dos rios Mundaú e Paraíba, respectivamente Santana do Mundaú e Capela (Figura 1). Ambos apresentam baixo índice de desenvolvimento humano (IDH), taxas de analfabetismo em torno de $50 \%$ e são regiões endêmicas da esquistossomose $\mathrm{e}^{14,15}$.

Santana do Mundaú, com clima tropical chuvoso e vegetação de zona da mata, localiza-se na microrregião Serrana dos Quilombos no norte de Alagoas, na divisa com o Estado de Pernambuco ${ }^{15}$. O rio Mundaú corta a cidade, sendo responsável pela drenagem de boa parte dos esgotos domésticos. Mesmo contaminado, suas águas servem para lazer, atividades laborais e de uso doméstico para a população ribeirinha. A restrita área urbana do município, banhada em totalidade pelo rio Mundaú, dispõe de apenas uma ponte. Atravessar o rio torna-se uma constante, também como via de acesso à escola.

O município de Capela está localizado no centro-norte alagoano, na microrregião da Mata Alagoana. Seu clima é tropical chuvoso com verão seco. A vegetação de Mata Atlântica está quase destruída em função do plantio de cana-de-açúcar, retirada de madeira ou da agricultura de subsistência ${ }^{14}$.

\section{Metodologia do estudo de campo}

O município de Santana do Mundaú tem 2.730 alunos matriculados em escolas municipais e, em Capela, esse número sobe para $4.810^{14,15}$. Os critérios para inclusão no estudo foram a idade, o preenchimento dos termos de consentimento e dos inquéritos e a entrega das amostras fecais. Dessa forma, o grupo de estudo foi constituído por 690 alunos com idade entre sete e 15 anos, provenientes de escolas públicas dos municípios estudados. Primeiramente, o projeto foi apresentado à comunidade e os termos de consentimento livre e esclarecido (TCLE) foram assinados pelos responsáveis. Foi aplicado um inquérito socioeconômico aos alunos, com questões sobre o tipo e condições de moradia, destino do lixo e dejetos, escolaridade e renda dos pais, hábitos, contato com coleções de água e conhecimento de moluscos transmissores.

Em outra etapa, foram distribuídos coletores universais para amostras fecais. Esse material foi recolhido, acondicionado em recipiente de isopor com gelo e levado ao Laboratório de Esquistossomose e Malacologia do Instituto de Ciências Biológicas e da Saúde (ICBS) da Universidade Federal de Alagoas (UFAL), onde foi mantido em ambiente refrigerado até a realização dos exames parasitológicos.

Após os resultados, foi providenciado o receituário para os casos positivos de esquistossomose e parasitoses intestinais. Os responsáveis pelos alunos foram orientados quanto ao tratamento. A apresentação de material educativo sobre a doença, meios de controle e conscientização ambiental foi realizada por meio de vídeos, palestras e cartazes, para alunos, familiares e funcionários.

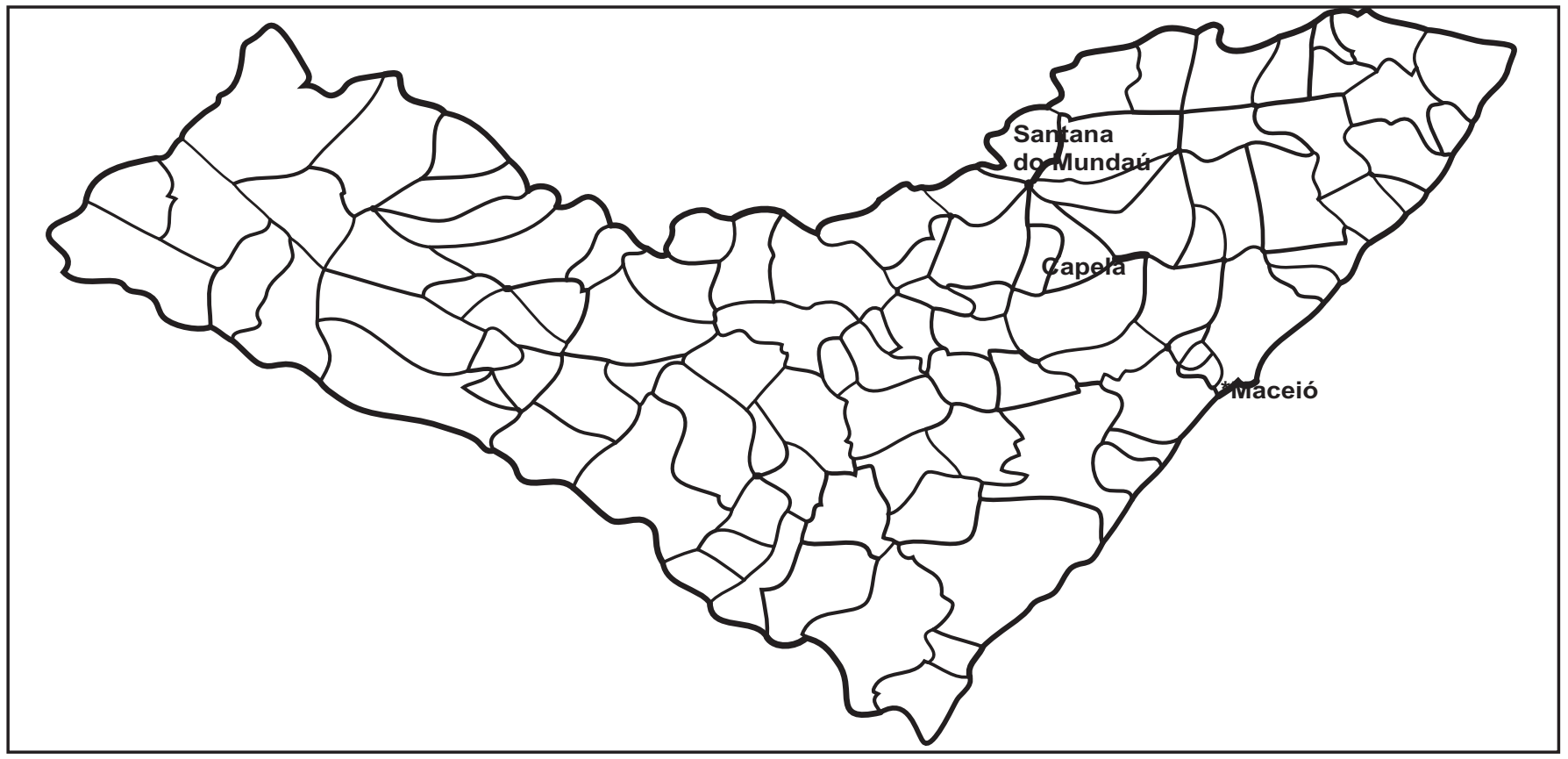

FIGURA 1 - Mapa do Estado de Alagoas com a localização dos municípios estudados. Fonte: www.santiagosiqueira.pro.br 


\section{Exames coproscópicos}

Foram examinados 690 escolares, dos quais 404 (58,6\%) eram procedentes de Santana do Mundaú e 286 (41,5\%) de Capela. As amostras fecais foram processadas dentro de 72 horas, através dos métodos de Lutz e de Kato, de acordo com as normas e orientações preconizadas ${ }^{4-6,16}$. Para cada material, foram preparadas duas lâminas, para análise em microscópio de luz por pesquisadores diferentes, para maior segurança do diagnóstico. Nos casos positivos para esquistossomose, foi realizada a contagem dos ovos.

\section{Análise estatística}

Utilizou-se o programa Epi-info Windows 3.5.1. Para análise dos questionários socioeconômicos e exames coproscópicos, observaram-se: frequências simples, análise de risco, qui quadrado e teste exato de Fisher, aplicados em situações específicas. Valores de $\mathrm{p} \leq 0,05$ foram considerados significantes. Para o estudo da relação entre os métodos parasitológicos, obtiveram-se o coeficiente Kappa e frequências simples.

\section{RESULTADOS}

Os exames parasitológicos de fezes positivos foram em número de 481 (69,7\%), com uma taxa de positividade para esquistossomose de $24,9 \%\left(n^{\circ}=172\right)$. Houve uma maior prevalência em Santana do Mundaú com 112 crianças infectadas (27,7\% do total local). Porém, Capela também demonstrou elevados valores, com 60 escolares infectados (20,9\%).

Dentre osinfectados, 60(34,8\%) estavamacometidos exclusivamente pelo $S$. mansoni, enquanto os demais $112(65,1 \%)$ apresentaram coinfecção com outros helmintos ou protozoários. Como se observa na Tabela 1, houve significância na associação $S$. mansoni/Trichuris trichiura $(\mathrm{p}=0,03)$ e S. mansoni/protozoários $(\mathrm{p}=0,04)$.

A comparação entre os métodos coprológicos demonstra que a técnica de Lutz identificou maior número de positivos do que a de Kato (Tabela 2). Além disso, os métodos apresentam resultados sinérgicos em apenas $45,8 \%$ dos casos positivos.

A carga parasitária média encontrada foi de 151,4 opg, maior em Santana do Mundaú (187,9 opg) que em Capela (83,2 opg). Foram encontrados valores entre 24 e $4.584 \mathrm{opg}$, tendo como moda $24 \mathrm{opg}$.

TABELA 1 - Parasitoses intestinais em escolares dos municípios de Capela e Santana do Mundaú e sua associação com o $S$. mansoni no período de 2006-2008.

\begin{tabular}{|c|c|c|c|c|c|c|c|}
\hline \multirow[b]{2}{*}{ Parasitoses Intestinais } & \multicolumn{3}{|c|}{$\begin{array}{l}\text { Amostra total } \\
\mathrm{n}^{\mathrm{o}}=690\end{array}$} & \multicolumn{4}{|c|}{$\begin{array}{c}\text { Co-infecção com S. mansoni } \\
\qquad \mathrm{n}^{\mathrm{o}}=172\end{array}$} \\
\hline & $\mathrm{n}^{\circ}$ & $\%$ & IC & $\mathrm{n}^{\mathrm{o}}$ & $\%$ & RR & $\mathrm{p}$ \\
\hline Helmintos & 385 & 55,8 & $52-59$ & 172 & 100 & - & - \\
\hline A. lumbricoides & 188 & 27,2 & $24-31$ & 54 & 31,4 & $0,9-1,6$ & 0,16 \\
\hline Ancylostomidae & 60 & 8,7 & $7-11$ & 20 & 11,6 & $0,9-2,0$ & 0,11 \\
\hline E. vermicularis & 9 & 1,3 & $1-3$ & 2 & 1,2 & $0,3-3,0$ & 0,85 \\
\hline H. nana & 34 & 4,9 & $3-7$ & 6 & 3,5 & $0,3-1,5$ & 0,31 \\
\hline S. stercoralis & 12 & 1,74 & $1-3$ & 4 & 2,3 & $0,6-3,0$ & 0,5 \\
\hline T. trichiura & 178 & 25,8 & $23-29$ & 54 & 31,4 & $1,0-1,7$ & 0,03 \\
\hline Protozoários & 235 & 34,1 & $30-38$ & 49 & 28,5 & $0,6-1,0$ & 0,04 \\
\hline E. coli & 168 & 24,3 & $21-28$ & 34 & 19,8 & $0,5-1,1$ & 0,05 \\
\hline E. histolytica & 114 & 16,5 & $14-20$ & 22 & 12,8 & $0,5-1,1$ & 0,13 \\
\hline G. lamblia & 50 & 7,2 & $5-9$ & 11 & 6,4 & $0,5-1,5$ & 0,62 \\
\hline
\end{tabular}

IC: intervalo de confiança com $95 \%$, RR: risco relativo.
TABELA 2 - Estimativa da eficácia entre os métodos do Kato e de Lutz.

\begin{tabular}{lccccc}
\hline Método & Exames Positivos \% & IC & Kappa & IC & p \\
\hline Kato & 69,4 & $57-80$ & $-0,36$ & $-0,48$ a $-0,25$ & 0,0018 \\
Lutz & 76,4 & $65-86$ & & & \\
\hline
\end{tabular}

$\mathrm{n}^{\circ}: 172$ exames positivos para S. mansoni, IC: intervalo de confiança com $95 \%$. Kappa: coeficiente de correlação intraclasse.

Entre os 690 alunos investigados, a idade média foi de 9,6 anos. As crianças com esquistossomose encontraram-se na faixa etária dos sete aos dez anos (65\%), com idade média de dez anos. A ocorrência da doença foi mais comum nos indivíduos do sexo masculino, porque entre os 172 parasitados, 97 (56,4\%) eram meninos.

O inquérito mostrou que $50 \%$ dos alunos com esquistossomose residem em casa de alvenaria, os dejetos de suas casas são eliminados principalmente no sistema público de saneamento (44\%), existe coleta pública do lixo $(90,2 \%)$ e a água utilizada para consumo é obtida através da rede pública de abastecimento (76,9\%). A maior parte das crianças $(53,5 \%)$ conhece os moluscos transmissores, mas assegurou não frequentar o rio (Tabela 3 ).

TABELA 3 - Aspectos sócio-econômicos das crianças portadoras de esquistossomose dos municípios de Capela e Santana do Mundaú no período de 2006 - 2008.

\begin{tabular}{|c|c|c|c|c|}
\hline \multirow[b]{2}{*}{ Variáveis do Inquérito } & \multicolumn{2}{|c|}{$\begin{array}{l}\text { Amostra em geral } \\
\qquad n^{\circ}=269^{*}\end{array}$} & \multicolumn{2}{|r|}{$\begin{array}{l}\text { S. mansoni } \\
\qquad \mathrm{n}=60^{* *}\end{array}$} \\
\hline & $\%$ & IC & $\%$ & $\mathrm{p}$ \\
\hline \multicolumn{5}{|l|}{ Domicílio } \\
\hline bloco & 3,2 & $1-6$ & 0 & não é válido*** \\
\hline lajota & 27 & $21-33$ & 36 & \\
\hline madeira & 5,9 & $3-10$ & 6 & \\
\hline taipa & 7,7 & $4-12$ & 8 & \\
\hline alvenaria & 56,3 & $49-63$ & 50 & \\
\hline \multicolumn{5}{|l|}{ Destino de dejetos } \\
\hline céu aberto & 18,9 & $14-25$ & 22 & não é válido*** \\
\hline esgoto & 47,1 & $40-54$ & 44 & \\
\hline fossa & 33,9 & $28-40$ & 34 & \\
\hline \multicolumn{5}{|l|}{ Abastecimento d'água } \\
\hline clandestina & 4,8 & $2-8$ & 3,8 & não é válido*** \\
\hline poço & 17 & $12-22$ & 19,2 & \\
\hline rede pública & 78,2 & $72-83$ & 76,9 & \\
\hline \multicolumn{5}{|l|}{ Frequência no rio } \\
\hline $\operatorname{sim}$ & 46,5 & $40-53$ & 48,1 & 0.79 \\
\hline não & 53,5 & $47-60$ & 51,9 & \\
\hline \multicolumn{5}{|l|}{ Caramujos no rio } \\
\hline $\operatorname{sim}$ & 54,8 & $48-61$ & 55,8 & 0.87 \\
\hline não & 45,2 & $39-52$ & 44,2 & \\
\hline
\end{tabular}

*Amostra composta por indivíduos com exame coproscópico negativo ou parasitados apenas por S. mansoni. ${ }^{* *}$ Amostra composta por indivíduos parasitados apenas por S. mansoni sem associação com outras parasitoses. ${ }^{* * *} \mathrm{O}$ valor do Qui-quadrado não é válido. IC: intervalo de confiança com 95\%. RR: risco relativo.

\section{DISCUSSÃO}

Em 2008, foi referido que a esquistossomose, no Estado de Alagoas, estaria com uma prevalência de $8,54 \%$. Para o município de Capela, o índice seria de 22,8\% e para Santana do Mundaú, de $10,1 \%{ }^{17}$. Esses achados, apesar de demonstrarem percentuais altos, não condizem com os deste estudo, visto que aqui se encontrou uma maior prevalência em Santana do Mundaú (27,7\%). 
Segundo informações da Secretaria Estadual de Saúde de Alagoas (SESAU), os exames realizados por essa entidade são feitos por amostragem, ou seja, os coletores universais para amostras fecais são distribuídos aleatoriamente a uma parcela populacional de um bairro, viciando de certa forma o resultado. Acrescente-se ainda que, em alguns locais próximos a coleções de água, onde vivem populações humildes, as taxas de infecção humana chegam a $100 \%$.

No Brasil, a faixa etária de maior prevalência da esquistossomose abrange dos seis aos 20 anos. O contato mais assíduo dos jovens com as águas em atividades recreativas pode explicar tal predisposição. Por isso, o segmento infanto-juvenil, dos sete aos 15 anos de idade, foi adotado como parâmetro operacional para as atividades de controle no Brasil ${ }^{3,12,18,19}$, e também investigado nesta pesquisa.

A maior predisposição do sexo masculino para a esquistossomose justifica-se pelo hábito cultural dos meninos. A observação do comportamento social da comunidade demonstra que os mesmos costumam ter atividades de lazer, como banhos, pesca e práticas esportivas, acompanhados ou não dos pais, com mais frequência que as meninas ${ }^{13}$.

Em Santana do Mundaú, o problema acentua-se porque os escolares se vêem obrigados a atravessar o rio para frequentar as aulas, em constante exposição ao parasito. Além disso, algumas famílias represam a água para banhos caseiros ou uso doméstico, como lavagem de roupa ou louça.

A Organização Mundial de Saúde (OMS) considera como alta uma prevalência acima de $5 \%^{1}$ e, por outro lado, a elevada prevalência aqui encontrada foi também duas vezes maior que a apresentada pelo Programa de Controle da Esquistossomose ${ }^{17}$. Desse modo, Santana do Mundaú e Capela estariam caracterizadas como zonas hiperendêmicas. Os dois municípios exigem investimentos urgentes em infra-estrutura, educação em saúde, saneamento básico e medidas preventivas, através de uma atuação em conjunto com prefeitura, órgãos de saúde pública e toda a sociedade.

As infecções por parasitos intestinais estão frequentemente associadas; no entanto, poucos estudos informam a esse respeito, o que dificulta a interpretação e comparação dos resultados ${ }^{20}$. A relação entre $S$. mansoni e parasitos intestinais como Trichiurus trichiura e protozoários, observada aqui, necessita de maior investigação.

Para Lemos e cols, o emprego de técnicas simultâneas leva à obtenção de melhores esultado ${ }^{21}$. Mendes e cols afirmam que, como rotina em laboratório, utiliza-se mais de um método coproscópico para a detecção de formas parasitárias de protozoários e helmintos, principalmente em amostras com baixa carga parasitária. A diferença de resultados entre as duas técnicas demonstra que um indivíduo não deve ser classificado como sadio pelo resultado negativo de um método isolado ${ }^{22}$.

Exames para o diagnóstico da esquistossomose nem sempre se ajustam ${ }^{23}$. Rabello e cols realizaram testes imunológicos em pacientes com suspeita de esquistossomose. Foram 217 os casos confirmados; no entanto, quando se efetuaram exames coprológicos, verificouse apenas $68 \%$ de positividade. Tal fato demonstra uma baixa sensibilidade dos métodos diagnósticos utilizados. Ocorrência que pode ser revertida pelo aumento do número de amostras examinadas, uma vez que os métodos coproscópicos são equivalentes ${ }^{24}$.

As endemias específicas de vários municípios nordestinos, como Santana do Mundaú e Capela, abrangem aspectos ambientais, sociais, políticos e culturais. Medidas de engenharia sanitária são urgentes, entre outras. Neste trabalho, tornou-se essencial a etapa educacional. O científico aliado ao lúdico dos filmes, palestras, aulas expositivas e peças teatrais, foi de grande relevância para a conscientização dos escolares, pais e professores.

A educação continuada pode conduzir a uma mudança de hábitos nas crianças. O binômio educação em saúde e medidas técnicas de controle tem ação comprovadamente profilática e paliativa, diminuindo os índices de reinfecção, as cargas parasitárias e o aparecimento da forma hepatoesplênica ${ }^{1,3,12}$.

Endemia urbana importante, este estudo aponta para a necessidade de medidas emergenciais. Um maior investimento em melhorias sanitárias, a mobilização e participação da comunidade para educação em saúde e a constante atualização do perfil epidemiológico são a base para o remodelamento da esquistossomose no estado.

\section{AGRADECIMENTOS}

Ao Prof. Jairo Calado pela análise estatística. Ao técnico de laboratório Sr. João Florentino dos Santos, pelo apoio e orientações na realização dos exames parasitológicos de fezes. Às Secretarias Municipais de Saúde e Educação dos municípios pelo suporte.

\section{CONFLITO DE INTERESSE}

Os autores declaram não haver nenhum tipo de conflito de interesse.

\section{REFERÊNCIAS}

1. World Health Organization [Internet]. Schistosomiasis disease information. 2008 [acesso 17 de Julho de 2008]. Disponível em: http://www.who.int/tdr/ diseases/schisto/diseaseinfo.htm.

2. Rey L. Epidemiologia e controle da esquistossomose nas Américas. In: Bases da Parasitologia Médica. Rio de Janeiro: Ed. Guanabara-Koogan; 1992. p. 160170

3. Melo AL, Coelho PMZ. Schistosoma mansoni e a doença. In: Neves DP, editor. Parasitologia Humana. $11^{\text {th }}$ ed. Rio de Janeiro: Editora Atheneu; 2005. p. 193212

4. Moraes RG, Leite CI, Enio EG. Exame parasitológico de fezes. In: Parasitologia e Micologia Humana. $4^{\text {th }}$ ed. Rio de Janeiro: Ed. Cultura Médica; 2000. p. 681697

5. De Carli GA. Exames macroscópicos da amostra fecal fresca e preservada. In: De Carli GA, editor. Parasitologia Clínica. 1 $^{\text {st }}$ ed. São Paulo: Ed. Atheneu; 2001. p. 27-81.

6. Katz N, Chaves A, Pellegrino J. A simple device for quantitative stool thick-smear technique in Schistosomiasis mansoni. Rev Inst Med Trop 1972;14:397-400.

7. Fundação Nacional da Saúde (FUNASA). Programa de Controle da Esquistossomose. Relatório de Atividades; 1995.

8. Couto JLA. Biomphalaria (Mollusca: Pulmonata: Basommatophora) nas Meso-Regiões do Agreste e do Leste Alagoano, Alagoas, Brasil. Taxonomia, considerações ecológicas e epidemiológicas. Monografia. Universidade Federal de Alagoas; 1995.

9. Couto JLA. Estudo e identificação de espécies de Biomphalaria transmissores de esquistossomose em três municípios do Estado de Alagoas. XV Congresso Brasileiro de Parasitologia. Salvador; 1997.

10. Couto JLA. Esquistossomose mansoni em duas mesorregiões do Estado de Alagoas. Rev Soc Bras Med Trop 2005; 38:301-304.

11. Rocha RS, SilvaJG, Peixoto SV, Caldeira RL, FirmoJOA, Carvalho OS, etal. Avaliação da esquistossomose e de outras parasitoses intestinais em escolares do município de Bambuí, Minas Gerais, Brasil. Rev Soc Bras Med Trop 2000; 33:431-436. 
12. Castillo O. La promoción del desarrollo y la salud de los niños através del control de las helmintiasis. In: Reunión sobre el Control de las Helmintiasis Intestinales en el Contexto de AIEPI. Rio de Janeiro; 1998. p. 28-29.

13. Guimarães ICS, Tavares-Neto J. Transmissão urbana de esquistossomose em crianças de um bairro de Salvador, Bahia. Rev Soc Bras Med Trop 2006; 39:451455 .

14. Ministério de Minas e Energia. Projeto Cadastro de Fontes de Abastecimento por Água Subterrânea, estado de Alagoas: Diagnóstico do Município de Capela [Internet]. Recife: CPRM/PRODEEM; Agosto 2005 [acesso 17 de Julho de 2008]. 20 p. Disponível em: http://www.cprm.gov.br/rehi/atlas/alagoas/ relatorios/CAPE019.pdf.

15. Ministério de Minas e Energia. Projeto Cadastro de Fontes de Abastecimento por Água Subterrânea, estado de Alagoas: Diagnóstico do Município de Santana do Mundaú [Internet]. Recife: CPRM/PRODEEM; Agosto 2005 [acesso 17 de Julho de 2008]. 19 p. Disponível em: http://www.cprm.gov.br/rehi/atlas/ alagoas/relatorios/SDMU089.pdf.

16. Lutz A. O Schistosoma mansoni e a schistosomose segundo observações feitas no Brasil. Mem Inst Oswaldo Cruz 1919; 11:121-155.

17. Ministério da Saúde. Programa de Controle da Esquistossomose - PCE [Internet]. Acesso 11 de Outubro de 2009. Disponível em: http://tabnet.datasus. gov.br/ cgi/deftohtm.exe?sinan/pce/cnv/pce.def.

18. Ferreira UM, Ferreira CS, Monteiro CA. Tendência secular das parasitoses intestinais na infância na cidade de São Paulo (1984-1996). Rev Saude Publica $2000 ; 34: 1-16$

19. Katz N. Las helmintiases a nivel mundial: situación actual. In: Reunión sobre el control de las helmintiases intestinales en el contexto de AIEPI. Rio de Janeiro; 1998. p.13-16

20. Brooker S, Alexander N, Geiger S, Moyeed RA, Stander J, Fleming F, et al. Contrasting patterns in the small-scale heterogeneity of human helminth infections in urban and rural environments in Brazil. Int $j$ Parasitol 2006; 36: 1143-1151.

21. Lemos CPA, Lima DMC, Silva LC, Chieffi PP. Parasitological diagnosis of schistosomiasis mansoni: concomitant utilization of Kato-Katz method and hatching test. Rev Inst Med Trop Sao Paulo 1995; 37:471-472.

22. Mendes CR, Teixeira ATLS, Pereira RAT, Dias LCS. Estudo comparativo de técnicas parasitológicas: Kato-Katz e coprotest ${ }^{\circledR}$. Rev Soc Bras Med Trop 2005, 38:178-180.

23. Santos FLN, Cerqueira EJL, Soares NM. Comparison of the thick smear and Kato-Katz techniques for diagnosis of intestinal helminth infections. Rev Soc Bras Med Trop 2005; 38:196-198.

24. Rabello ALT, Rocha RS, Oliveira JPM, Katz N, LambertucciJR. Stool examination and rectal biopsy in the diagnosis and evaluation of therapy of schistosomiasis mansoni. Rev Inst Med Trop Sao Paulo 1992; 34: 601-608. 